\title{
Searching for planetary nebulae at the Galactic halo via J-PAS
}

\author{
Denise R. Gonçalves ${ }^{1}$, T. Aparício-Villegas ${ }^{2,3}$, S. Akras ${ }^{1}$, A. Cortesi ${ }^{4}$, \\ M. Borges-Fernandes ${ }^{2}$, S. Daflon ${ }^{2}$, C. B. Pereira ${ }^{2}$, S. Lorenz-Martins ${ }^{1}$, \\ W. Marcolino ${ }^{1}$, A. Kanaan ${ }^{5}$, K. Viironen ${ }^{6}$, C. Mendes de Oliveira ${ }^{4}$, \\ A. Molino ${ }^{4}$ A. Ederoclite ${ }^{6}$ and the J-PAS Collaboration \\ ${ }^{1}$ Observatório do Valongo - Universidade Federal do Rio de Janeiro, Brazil; email: \\ denise@astro.ufrj.br ${ }^{2}$ Observatório Nacional - Ministério de Ciência e Tecnologia, Brazil; \\ ${ }^{3}$ Instituto de Astrofísica de Andalucía, Spain ${ }^{4}$ IAG - Universidade de São Paulo, Brazil; \\ ${ }^{5}$ Departamento de Física - Universidade Federal de Santa Catarina, Brazil; ${ }^{6}$ Centro de \\ Estudios de Física del Cosmos de Aragón, Spain.
}

\begin{abstract}
The Javalambre-Physics of the Accelerating Universe Astrophysical Survey (J-PAS) is a narrow-band imaging, very wide field cosmological survey. It will last 5 years and will observe 8500 sq. deg. of the sky. There will be 54 contiguous narrow-band filters of $145 \AA$ FWHM, from 3,500 to $10,000 \AA$. Two broad-band filters will be added at the extremes, UV and IR, plus the $3-g$, $r$, and $i$-SDSS filters. Thus, J-PAS can be an important tool to search for new planetary nebulae $(\mathrm{PNe})$ at the halo, increasing their numbers, because only 14 of them have been convincingly identified in the literature. Halo PNe are able to reveal precious information for the study of stellar evolution and the early chemical conditions of the Galaxy. The characteristic low continuum and intense emission lines of PNe make them good objects to be searched by J-PAS. Though covering a significantly smaller sky area, data from the ALHAMBRA survey were used to test our J-PAS strategy to search for PNe. Our first results are shown in this contribution.
\end{abstract}

\section{Halo PNe properties versus J-PAS magnitudes}

Halo PNe like BoBn 1, DdDm 1 and PS 1 are located somewhere between 11 and $24 \mathrm{kpc}$ from the Sun, and have B magnitudes of 16, 14 and 13.4, respectively (Howard et al. 1997). Such values are easily encompassed by the J-PAS limits (Benítez et al. 2014).

We have used the J-PAS narrow-band photometry to study the possibility of detecting PNe (Benítez et al. 2014). From the observed spectra of known halo PNe, we got the J-PAS synthetic photometry. In Fig. 1 we show the observed spectrum of a halo PN (top panel), as well its J-PAS "SED" (in magnitudes; bottom-left panel), in which it is straightforward to see the two conspicuous emissions (associated with the intense [O III] and $\mathrm{H}_{\alpha}$ emission lines). Therefore, halo PNe can easily be detected by the J-PAS survey.

\section{ALHAMBRA: PNe in the Galactic halo or in nearby galaxies?}

Using the average characteristics of halo PNe, we constructed a grid of CLOUDY models to simulate the spectra of these objects. With these simulated spectra, other 4 observed spectra (MWC 574, Pereira \& Miranda 2007; DdDm 1, Kwitter et al. 1998; NGC 2242, Kwitter et al. 2003; and H 4-1, SDSS, respectively), and the Viironen et al. (2009) colour-colour diagram, we defined the location where halo PNe are better separated from other emission line objects, in the $r^{\prime}-H_{\alpha}$ versus $r^{\prime}-i^{\prime}$ plane.

Though covering a significantly smaller sky area, data from the ALHAMBRA survey (Moles et al. 2008) were used to test our strategy. The ALHAMBRA photometric system (Aparıcio-Villegas et al. 2010) consists of 20 optical medium-band filters, plus the 

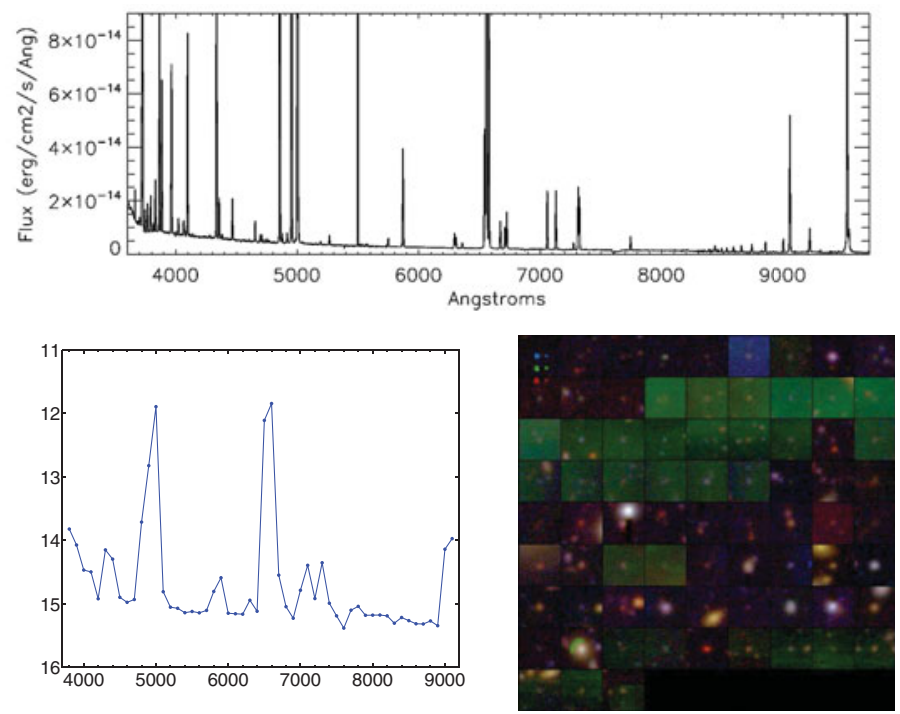

Figure 1. Top: spectrum of DdDm 1 (Kwitter et al. 1998). Bottom-left: J-PAS "SED" based on this spectrum. Bottom - right: composite (Subaru-B, Subaru-R and F814-HST) images of the selected PN candidates.

three standard $J H K$ s near-infrared (NIR) bands. Because of the availability of the NIR magnitudes, the Viironen et al. (2009) near-IR $\left(J-H\right.$ versus $H-K_{s}$, left panel of their Fig. 1) diagram was also used to better define the PN candidates. Using the optical diagram we identified 75 possible PN candidates in the ALHAMBRA fields, number which was significantly reduced by using the NIR plane. Candidates found this way were then filtered by having low ODDS (that measures how well a source fits to a template of any kind of galaxy; see Molino et al. 2014). This fact should help eliminating high-redshift galaxies from the candidate list (in ALHAMBRA the galaxy distribution peaks at $\mathrm{z}=0.3$, with only a few reaching $\mathrm{z}=1$ ). High-z ( 3.1 to 3.3 ) AGNs and QSOs, with Ly $\alpha 1216 \AA$ and CIV $1549 \AA$ mimicking the [O III] and $\mathrm{H}_{\alpha}$ emission-lines, could appear among our candidates (Ciardullo et al. 2002) - we are testing other colour-colour diagrams, for JPAS, to avoid these high-z impostors. We ended up with 16 bona-fide ALHAMBRA PN candidates. Their nature is being confirmed via follow-up spectroscopy (proposals were submitted for 2016A). The candidates we identified are faint (from 19 to 24 in V mag), therefore, instead of halo PNe, they are most probably extragalactic PNe. If proposals are successful, results will be available before the start of the J-PAS survey.

\section{References}

Aparicio-Villegas, T., Alfaro, E. J., Cabrera-Caño, J., Moles, M., et al. 2010, AJ, 139, 1242

Benítez, N., Dupkeb, R., Moles, M., Sodré, L., Cenarro, A. J., et al. 2014, astro-ph/, arXiv, 1403.5237

Ciardullo, R, Feldmeier, J. J., Krelove, K., et al. 2002, ApJ, 566, 784

Howard, J. W., Henry, R. B. C., \& McCartney, S. 1997, MNRAS, 284, 654

Kwitter K. B. \& Henry R. B. C. 1998, ApJ, 493, 247

Kwitter, K. B., Henry, R. B. C., \& Milingo, J. B. 2003, PASP, 115, 80

Moles M., Benítez N., Aguerri J. A. L., Alfaro E. J., Broadhurst T., et al. 2008, AJ, 136, 1325

Molino A., Benítez N., Moles M., et al. 2014, MNRAS,441, 2891

Pereira, C. B. \& Miranda, L. F. 2007, $A \& A$, 467, 124

Viironen, K., Mampaso, A., Corradi, R. L. M., Rodríguez, M., et al. 2009, A\&\&A, 502, 465 